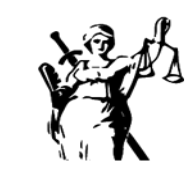

JUSTICIA

ISSN impreso 0124-7441
ISSN digital 2590-4566

\title{
Definición de Perfiles Geográficos de hurto de automóviles. Caso Aplicado en Cartagena de Indias.
}

\section{Definition of Geographic Profiles of car theft. Applied case in Cartagena de Indias.}

\author{
(iD) \\ Enrique Jose Delahoz-Dominguez \\ Universidad Tecnológica de Bolívar, Colombia \\ edelahoz@utb.edu.co
}

\author{
Tomás José Fontalvo-Herrera \\ Universidad de Cartagena, Colombia \\ tfontalvoh@unicartagena.edu.co
}

\begin{abstract}
(iD)
Adel Alfonso Mendoza-Mendoza

Universidad del Atlántico, Colombia

adelmendoza@uniatlantico.edu.co

Recibido: 07 de noviembre de 2019 / Aceptado: 11 de febero de 2020

https://doi.org/10.17081/just.25.37.4215

Resumen
\end{abstract}

Esta investigación desarrolla un análisis geoespacial del hurto de automóviles ocurridos en la ciudad de Cartagena de Indias - Colombia. En el proceso de definición de perfiles de criminalidad se analizó el delito del hurto en las 5 modalidades consideradas de alto impacto en la generación de percepción de seguridad de los ciudadanos, estas son automotores, motocicletas, residencias, establecimientos comerciales y entidades financieras en el transcurso de los años 2015 y 2016. Los datos utilizados corresponden al informe de criminalidad anual de la Policía Nacional de Colombia. Como resultado se encontraron 7 perfiles geográficos de hurto de automóviles, generando patrones de delitos caracterizados visualizados geográficamente.

PALABRAS CLAVE: SMEs, machine learning, decision-making, management.

\begin{abstract}
In the present research a geospatial analysis of the crimes occurred in the city of Cartagena de Indias in Colombia is developed. In the process of definitions of crime profiles, the crime of theft is analyzed in the 5 Modalities considered to have a high impact on the generation of security perception of Citizens, which are motor vehicles, motorcycles, residences, commercial establishments and financial entities during the course of the years 2015 and 2016. The data used corresponds to the annual crime report of the National Police of Colombia. As a result, 7 geographical profiles of car theft were found, generating a patron of characterized crimes displayed geographically.
\end{abstract}

KEYWORDS: PYMES, aprendizaje automático, toma de decisiones, gestión.

\section{Como citar:}

Delahoz-Dominguez, E. J., Fontalvo-Herrera, T. J., \& Mendoza-Mendoza, A. A. (2020). Definición de Perfiles Geográficos de hurto de automóviles. Caso Aplicado en Cartagena. Justicia, 25(37), 99-108. https://doi.org/10.17081/just.25.37.4215 


\section{Introduction}

La presente investigación desarrolla la definición de perfiles geográficos de hurto de vehiculos en Cartagena, mediante la aplicación de la técnica de minería de datos. El objetivo planteado es analizar y describir los patrones y tendencias geoespaciales identificados en los hurtos a automóviles cometidos en la ciudad durante los años 2015-2016, teniendo en cuenta los registro obtenidos del portal del gobierno colombiano para la publicación de datos públicos www.datos.gov.co. Todo esto enfocado en la percepción de Cartagena como una ciudad turística, con alto reconocimiento internacional, lo cual implica que el incremento de este tipo de actos delictivos no solo afecta la buena imagen de la ciudad como destino turístico de clase mundial, sino también repercute en el bienestar de la población nativa, quienes diariamente se encuentran expuestos a los riesgos asociados a la inseguridad ciudadana.

De esta investigación se propone un modelo replicable y extrapolable, que sirva como fuente de información y sea la base para el desarrollo de futuras investigaciones para el análisis de la seguridad en otras ciudades. Así mismo, se busca generar un conocimiento en los ciudadanos a través de la presentación geográfica de los resultados. Lo anterior permite consolidar el enfoque visual como una realización de la experiencia, considerando los conceptos y su representación como algo indivisible (Fontalvo-Herrera et al., 2018).

En la primera parte del desarrollo de artículo se muestra una panorámica general de los hurtos a nivel global y local, a fin de contextualizar a la ciudad de Cartagena con respecto a otras ciudades del mundo. Luego, se plantea el problema con los respectivos objetivos de la investigación. Seguidamente, se explica la metodología que se llevó acabo, exponiendo cada uno de los pasos que se ejecutaran durante el desarrollo del estudio. Posteriormente se describen diferentes investigaciones donde han hecho uso de la técnica de minería de datos para el análisis de grandes bases de datos sobre criminalidad, convirtiéndose en fuente de información para el desarrollo de nuestro proyecto (Fajardo Pascagaza, 2016; Ripoll, 2015; Soto, 2017). Posteriormente, se enuncia el planteamiento de la metodología de la investigación, la cual hace referencia a la implementación de la técnica de minería datos como solución viable para el análisis del comportamiento de los hurtos de automóviles. Finalmente, en análisis de resultados se plantean las conclusiones observadas y obtenidas a través del mapa geoespacial con respecto a los clústeres derivados de la investigación.

\section{Contexto global}

Los hurtos constituyen una de las problemáticas principales de seguridad a nivel mundial debido a su alta frecuencia y al impacto social que generan, llegando hasta el punto de convertirse en una de las mayores preocupaciones sociales ya que repercuten no solo en el patrimonio de la población, sino también sobre el capital social, bienestar y confianza de los ciudadanos; en este sentido se destaca que la violencia en América Latina está posicionada entre las principales cinco causas de muerte de la población, siendo la principal en Brasil, Colombia, Venezuela, El Salvador y México (Güitron \& Guerrero, 2017).

El alza del fenómeno de los hurtos a nivel mundial es significativa y de acuerdo con los indicadores oficiales en América Latina 28 millones de familias son sujetas a hurto o robo en un año, lo que equivale a 54 familias que son robadas por minuto (Londoño \& Guerrero, 1999). A partir de esto, se resaltan los siguientes

hallazgos referentes a América Latina: el problema de los robos se ha extendido y el uso de la violencia ha aumentado significativamente. 
Es así como, se han desarrollado mercados de artículos robados y existe un porcentaje de robos que no se denuncian por parte de las victimas (Félix, Ponce, Hernández, \& Llamas, 2015). De tal modo, los hurtos se han catalogado a nivel global como un acto cotidiano debido a su alta frecuencia de ocurrencia. Un aspecto alarmante es el uso de armas de fuego o armas blancas para efectuar los hurtos, por lo cual en algunas situaciones se presentan heridos y en las peores circunstancias ocurren casos de homicidio. En otras circunstancias, Argentina presenta una alta tasa de intimidación por arma de fuego en los robos, superior al $70 \%$, en cambio países como Chile y el Salvador no superan el $40 \%$. Llama la atención también que la mitad de los robos conocidos por los internos en Argentina se efectuaron con violencia; $39 \%$ en México, 37\% en Chile, 30\% en Perú y Brasil, y 12\% en El Salvador (Cubel, 2016). Ahora bien, en Nicaragua, según el estudio realizado por el Instituto de Estudios Estratégicos y políticas públicas (IEEPP), los robos encabezan la lista de los problemas de seguridad, con un $63.7 \%$, siendo los robos a casas y comercios la problemática principal. Según el Ministerio de Gobernación junto con las Naciones Unidas, es notoria la participación de jóvenes de entre 18 y 25 años en actividades criminales quedando detenidos por el 54\% de los robos con fuerza y el $67,5 \%$ de los robos con intimidación (Gutiérrez, 2013).

Por otra parte, España aparece como el tercer país de la Unión Europea que sufre mayor número de robos en las entidades comerciales, presentando cifras mayores al diez por ciento de la media comunitaria en robos a establecimientos de comercio y personas. Las pérdidas provocadas por los robos en este país superan más de 2.400 millones de euros (Ripollés, 2006). Por su parte, Guatemala en el último año presentó un elevado nivel de criminalidad; además al estar clasificada como una región de riesgo extremo junto a México y Centroamérica, la criminalidad le ha costado unos US\$200.000 millones debido a los hurtos y a las personas fallecidas como consecuencia de la falta de seguridad (Abullarade, 2016).

Otro país que amerita ser resaltado es Brasil, en el cual según el Instituto de Seguridad Pública de Río de Janeiro se ha registrado un aumento del número de robos y asaltos en las calles, que subieron un 23,7 $\%$, hasta 38.461 casos entre enero y abril de 2016. Igualmente se registró un crecimiento del $19,7 \%$ en el número de robo de vehículos, hasta la cifra de 13.074 unidades (Dammert \& Arias, 2007). Finalmente, estas estadísticas conducen a interpretar que los análisis relacionados con los registros de los hurtos no han sido efectivos para la generación de políticas y planes de prevención de este fenómeno global debido a que los indicadores presentan tenencias alcistas significativos, por lo tanto se necesitan herramientas que permitan tomar decisiones operativas para el mejoramiento de la seguridad ciudadana.

\section{Contexto local}

Cartagena, es una ciudad histórica, caracterizada por sus calles coloniales, llenas de magia y colorido. Lo que la ha posicionado como un destino privilegiado para el turismo, la cultura, los eventos negocios de talla mundial y la inversión extranjera.

Según el ministerio de comercio, industria y turismo, en el período de Enero - Mayo del 2016, el sector turístico en la ciudad de Cartagena registró la llegada de 161.156 pasajeros por concepto de entrada de 109 cruceros, 12,37\% mayor que el mismo período del año anterior. Además, el número de viajeros que reportaron a Bolívar como destino principal creció de 131.008 a 162.423, posicionándose como el segundo departamento con mayor demanda turística en el país por parte de no residentes, después de la ciudad de Bogotá. No obstante, la ciudad continúa en la lucha por el progreso y el desarrollo sostenible, que se ha visto obstruido por los actos delictivos que se comente a diario en la ciudad, los cuales no generan una percepción de seguridad adecuada para la consolidación y prosperidad de la inversión local y extranjera. 
Es por esto, que la disminución de este tipo de actos como deben de ser una prioridad para las autoridades locales. Teniendo en cuenta que los hurtos en ocasiones no solo generan daños materiales, sino que también transcienden a homicidios causados en su mayoría por la resistencia que colocan los habitantes con el fin de salvaguardar sus bienes personales.

\section{Metodología}

Este artículo de investigación presenta un método de caracterización de delitos relacionados con hurtos en la ciudad de Cartagena, soportado por el desarrollo de técnicas de minería de datos (DM). Para el desarrollo de esta investigación se partió de información primaria suministrada por el gobierno. El desarrollo central de la investigación está basado en un modelo de aprendizaje no supervisado de datos, implementando las técnicas de análisis de componentes principales y el método de clustering en los datos. El trabajo de investigación se centró en el desarrollo de dos etapas: la reducción de dimensiones de las variables estudiadas

y la creación de escenarios de hurtos. El proceso de reducción de dimensiones crea un conjunto de nuevas variables no correlacionadas permitiendo una interpretación visual del resultado final. Como valor agregado se aportó un método para caracterizar los hurtos que permite identificar características asociadas con los respectivos pesos de cada variable por grupo encontrado.

Cabe aclarar que, esta investigativo tiene un enfoque cuantitativo, que usa la recolección de datos para probar una hipótesis, con base a la medición numérica y el análisis estadístico de datos, para establecer patrones de comportamientos y probar teorías (Oates, 2005). Por consiguiente, mediante este tipo de investigación se desea explicar y predecir de manera objetiva la problemática social, relacionada con los hurtos de automóviles acontecidos en la ciudad de Cartagena en los años 2015 y 2016, buscando regularidades y relaciones causales entre los datos.

Además, se tiene como finalidad analizar una problemática social desde un punto de vista cuantitativo, implementando el método inductivo, apoyado en el estudio y análisis de la base de datos suministrada por el gobierno. Por lo que se parte, de una teoría o planteamiento del problema, basado en la observación de los datos relacionados con los actos delictivos ocurridos en la ciudad, para posteriormente, establecer propuestas, a través de la inferencia obtenida de los datos, la cual finalmente serán evidenciasdas. Para esto, se implementará la técnica de minería de datos, que nos permitirá extraer información sustancial del conjunto de datos con el fin de transformarla en una estructura comprensible.

\section{Metodología de caracterización de delitos}

Análisis del problema: El objetivo de esta fase consiste en examinar exhaustivamente el problema en cuestión que para este caso alude a varias modalidades de hurtos con ocurrencia en la ciudad de Cartagena durante los años 2015 y 2016. Así pues, el procedimiento inicial fue obtener información del problema con el fin de identificar detalladamente qué es lo que sucede y determinar las posibles causas que lo están generando, por tal motivo se procedió a realizar un estudio del contexto tanto global como local y de la situación actual que atraviesa la ciudad con relación a los niveles de delincuencia ligados a hurtos.

A partir de esto, se evidenció que a pesar de que en Cartagena existen registros sobre los distintos tipos de delitos que caracterizan la criminalidad, no siempre la población reporta efectivamente ante la autoridad competente las situaciones que la afectan y representan una amenaza para su seguridad, creando restricciones operativas para el buen funcionamiento del cuerpo policial en función dea garantizar 
la seguridad y buen vivir en la ciudad. Además, luego de la revisión documental se encontró que varias modalidades de hurtos tales como el de motocicletas o celulares han aumentado año tras año.

Datos: Los datos usados para el desarrollo de esta investigación han sido suministrados por una base de datos del Gobierno Colombiano dedicada exclusivamente a proveer esta información para investigar, desarrollar aplicaciones, crear visualizaciones e historias. De este modo, nos enfocamos en la búsqueda de los registros relacionados con delitos, específicamente en 4 modalidades de hurtos, hurto de automotores, hurto a residencias, hurto de motocicletas y hurto a comercios, centrándonos en los años 2015 y 2016 . Cabe resaltar que este estudio está limitado a los datos provenientes del gobierno, los cuales solo representan los delitos denunciados sin tener en cuenta los casos que no tienen registro formal de ocurrencia.

TABLA 1.

RESUMEN DE INVESTIGACIONES GEOESPACIALE DE CRIMINALIDAD

\begin{tabular}{|c|c|c|}
\hline Investigación & ¿Qué hizo? & Técnica \\
\hline $\begin{array}{l}\text { El análisis geográfico del delito y los } \\
\text { mapas de la delincuencia (Vázquez } \\
\text { González \& Soto Urpina, 2013) }\end{array}$ & $\begin{array}{l}\text { Se presentó una herramienta para el análisis de la delincuencia } \\
\text { urbana con base en la cartografía digital analizando los aspectos } \\
\text { geográficos de la delincuencia e identificando configuraciones y } \\
\text { tendencias entre datos }\end{array}$ & Cartografía digital \\
\hline $\begin{array}{l}\text { El robo de vehículos y su relación es- } \\
\text { pacial con el contexto sociodemográfi- } \\
\text { co en tres delegaciones centrales de la } \\
\text { Ciudad de México (Sánchez Salinas \& } \\
\text { Fuentes Flores, 2016). }\end{array}$ & $\begin{array}{l}\text { Se analizaron los patrones espaciales del delito de robo de } \\
\text { vehículos en la Ciudad de México, encontrando en los resultados } \\
4 \text { variables tales como el uso del suelo no residencial, la densidad } \\
\text { de población, los hogares con jefatura femenina y concentración } \\
\text { de población joven como factores que influyen en el robo de } \\
\text { vehículos. }\end{array}$ & $\begin{array}{l}\text { Modelo de regresión } \\
\text { lineal múltiple y análisis } \\
\text { exploratorio de datos } \\
\text { espaciales en específico el } \\
\text { Índice de Moran. }\end{array}$ \\
\hline $\begin{array}{l}\text { Modelación y predicción de focos de } \\
\text { criminalidad basado en modelos prob- } \\
\text { abilísticos (Flores Arias, 2014) }\end{array}$ & $\begin{array}{l}\text { Un método empírico para la generación automática de funciones } \\
\text { probabilísticas espaciales de riesgo, junto con un mecanismo } \\
\text { para la caracterización de la evolución temporal de los focos de } \\
\text { criminalidad. }\end{array}$ & Modelos probabilísticos \\
\hline $\begin{array}{l}\text { Modelo para la caracterización del } \\
\text { delito en la ciudad de Bogotá, apli- } \\
\text { cando técnicas de minería de datos } \\
\text { espaciales (Peña Suárez, 2017) }\end{array}$ & $\begin{array}{l}\text { Un modelo para caracterizar el comportamiento del delito } \\
\text { aplicando técnicas de minería de datos espaciales encontrando } \\
\text { tendencias y variaciones de comportamiento en los datos. }\end{array}$ & $\begin{array}{l}\text { Minería de datos espa- } \\
\text { ciales. }\end{array}$ \\
\hline $\begin{array}{l}\text { Predicción de crimen usando modelos } \\
\text { de Markov ocultos (Obrecht Ihl, 2014) }\end{array}$ & $\begin{array}{l}\text { Estudio para la prevención y análisis del crimen a partir de la } \\
\text { previsión situacional, utilizando las características del lugar y las } \\
\text { circunstancias que rodean la ocurrencia de un crimen para expli- } \\
\text { car los patrones observados. }\end{array}$ & Modelos de Markov \\
\hline $\begin{array}{l}\text { Generación del mapa de crimen del } \\
\text { municipio de Zipaquirá mediante } \\
\text { herramientas geoestadisticas y sig } \\
\text { (Rodriguez Rojas, 2017) }\end{array}$ & $\begin{array}{l}\text { Se analizó el crimen en el municipio de Zipaquirá aplicando técni- } \\
\text { cas geoestadisticas y de análisis espacial, generando mapas de } \\
\text { crimen y mostrando las relaciones espaciales de las variables. }\end{array}$ & $\begin{array}{l}\text { Herramientas geoesta- } \\
\text { disticas }\end{array}$ \\
\hline
\end{tabular}

Fuente: elaboración propia

Depuración de datos: En esta etapa, se implementa un proceso sistemático destinado a la limpieza de los datos que serán procesados en la investigación, con el objetivo de garantizar que estos sean exactos y se encuentran libre de errores. 
Todo lo anterior, enfocado en la adecuada gestión y análisis de los datos, buscando representar la realidad de la manera más precisa con información consistente a través de la detección y corrección oportuna de datos incorrectos, permitiendo caracterizar con mayor exactitud los patrones de hurtos de automóviles.

Clustering: Esta etapa se caracteriza por agrupar un conjunto de datos, sin tener clases predefinidas, tomando como base la similitud de los valores de los atributos de los distintos datos. Esta asociación, a diferencia de la clasificación, se realiza de forma no supervisada, ya que se desconoce de antemano las clases del conjunto de datos de entrenamiento.

Modelación: Para la creación del código se utilizará el lenguaje de programación de R Studio, como herramienta sustancial para el procesamiento y análisis de datos de este tipo de estudio, a través de la generación de informes, sustentados en gráficas.

Generación de conocimiento: Mediante el análisis y las conclusiones obtenidas de la investigación, se proporcionan unos perfiles geográficos de hurtos de vehículos, los cuales representan las tendencias del delito de manera estructurada.

\section{Revisión de la literatura}

Existen diversos estudios donde se desarrollan conceptos sobre la clasificación, análisis y estrategias para analizar problemas de criminalidad en entornos urbanos. Dentro de los trabajos analizados se pueden agrupar las diferentes líneas de pensamiento según su enfoque metodológico. Estas líneas conceptuales son las siguientes.

Desarrollos conceptuales basados en técnicas de análisis geoespacial: Estos estudios analizan los factores determinantes en la ocurrencia de hechos delictivos, involucrando variables espaciales asociadas al lugar de ocurrencia de los hechos (Ver tabla 1).

TABLA 2.

RESUMEN DE INVESTIGACIONES ESTADÍSTICAS SOBRE CRIMINALIDAD

\begin{tabular}{|c|c|c|}
\hline Articulo & ¿Qué hizo? & Técnica \\
\hline $\begin{array}{l}\text { Análisis de la percepción del hurto a } \\
\text { personas entre } 2012 \text { y } 2015 \text { en Bogotá } \\
\text { (Rojas Lopez \& Ramirez, 2017). }\end{array}$ & $\begin{array}{l}\text { Se realizó un análisis cuantitativo de la percepción de los ciu- } \\
\text { dadanos sobre el hurto a personas en la ciudad de Bogotá para } \\
\text { los periodos } 2012 \text { y 2015, analizando la cantidad de veces que una } \\
\text { persona es víctima de hurto. }\end{array}$ & Modelo logit-probit \\
\hline $\begin{array}{l}\text { Delincuencia y actividad económica } \\
\text { en México (Félix et al., 2015) }\end{array}$ & $\begin{array}{l}\text { Relación entre delitos y la actividad económica en México medi- } \\
\text { ante la estimación de ecuaciones de cointegración y ciclo común } \\
\text { para evaluar si las series comparten una tendencia. }\end{array}$ & $\begin{array}{l}\text { Econometría y series de } \\
\text { tiempo }\end{array}$ \\
\hline $\begin{array}{l}\text { Diagnóstico de seguridad/inseguri- } \\
\text { dad. Un estudio exploratorio en una } \\
\text { comunidad urbana (Sani \& Nunes, } \\
\text { 2016) }\end{array}$ & $\begin{array}{l}\text { Un estudio exploratorio desarrollado en una comunidad con altos } \\
\text { índices de delincuencia en la ciudad de Oporto mediante el uso de } \\
\text { un cuestionario. }\end{array}$ & Cuestionario \\
\hline $\begin{array}{l}\text { Determinación de los lugares de } \\
\text { mayor incidencia de delitos y violen- } \\
\text { cia en el Distrito Metropolitano de } \\
\text { Quito con base en técnicas estadísti- } \\
\text { cas espaciales (Vizuete Galeas, 2013). }\end{array}$ & $\begin{array}{l}\text { Delimitación del fenómeno delincuencial en tiempo, espacio, y } \\
\text { tipologías delictuales que aquejan a la ciudad de Quito para deter- } \\
\text { minar los llamados "hot spots" del crimen. }\end{array}$ & $\begin{array}{l}\text { Kriging, forma de inferen- } \\
\text { cia bayesiana }\end{array}$ \\
\hline
\end{tabular}

Fuente: elaboración propia 
Por otro lado, en la tabla 2 se encuentran los desarrollos conceptuales basados en análisis estadísticos: Estas investigaciones integran metodologías de análisis de casos, encuestas para aportar conocimiento sobre los factores asociados a la determinación de relacionas de causalidad para la ocurrencia de eventos delictivos.

\section{Resultados}

\section{Perfiles de hurto de automóviles}

En el análisis del clúster jerárquico se implementó el algoritmo PAM (Partition Around Medoids), en donde una observación central de los datos se relaciona con el objeto que minimiza la suma de la disimilaridad media entre los miembros de un mismo grupo (De-La-Hoz, De-La-Hoz, \& Fontalvo, 2019). Se obtuvieron los miembros representativos de cada clúster basados en los medoides, consecuentemente el análisis del plano geoespacial en dos dimensiones, permitió determinar perfiles de criminalidad a través de zonas características de hurto de vehículos (ZCHV) (Ver Figura 1), las cuales se describen a continuación.

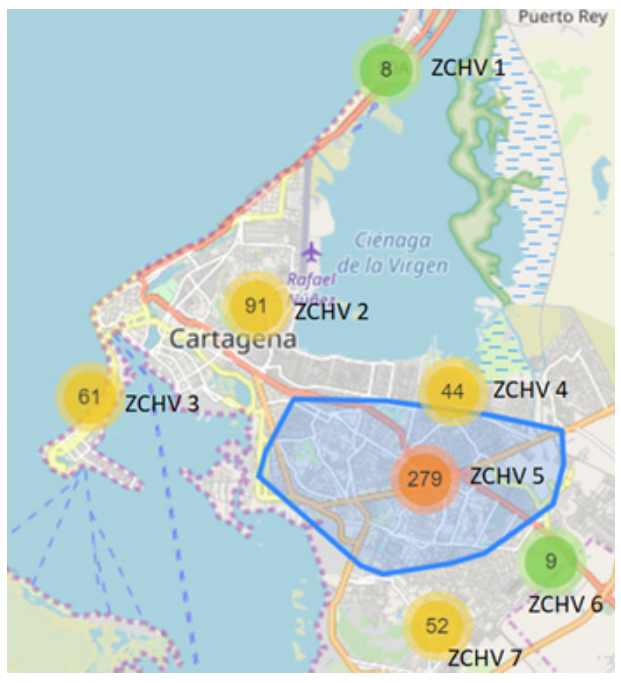

Figura 1. Clúster geográfico de hurto de automóviles

ZCHV 1: Representa la zona de acceso a la ciudad por la vía que comunica con la ciudad deBarranquilla, en el análisis se encuentran que en el periodo de estudio se presentaron 8 hurtos de vehículos, todos estos desarrollados utilizando arma de fuego y efectuados en la franja horario de 10 de la noche a 6 de la mañana. En cuanto a la clase del bien hurtado, se encuentra que 5 correspondían camionetas y 3 a vehículos.

ZCHV 2: Representa la zona del centro histórico de la ciudad, donde están algunos de los sitios emblemáticos de mayor visita por parte de los turistas. Se presentaron allí 91 hurtos, en donde prevalece el uso de llaves maestras (79\%) como herramienta principal para cometer el delito y el uso de herramientas alternativas como la escopolamina (9 \%). En la variable franja horaria no se identifica un patrón determinado, sin embargo, es válido mencionar que el $61 \%$ de los hurtos ocurre en la franja horaria comprendida entre las 6 de la tarde y las 5 de la mañana. Es importante aclarar que en esta zona se encuentra prohibido la circulación de motocicletas, por lo cual se explica que el $93 \%$ de los hurtos correspondan a vehículos automotores.

ZCHV 3: Representa la zona hotelera en el sector costero de la ciudad, aquí se localizan las grandes ca- 
denas hoteleras y es una de las zonas con el valor por metro cuadrado más alto de la ciudad.

En esta zona se presentarón 61 hurtos, prevaleciendo el uso de llaves maestras (88\%) como herramienta principal para cometer el delito. El análisis de los días de ocurrencia muestra como en los fines de semana se presenta el 73\% de los hurtos, además se destaca que el $69 \%$ de los vehículos hurtados correspondían a modelos superiores al año 2013. Es válido anotar que esta zona presenta una restricción para la circulación de motocicletas las 24 horas del día.

ZCHV 4: Representa la zona aledaña al sector de la ciénaga de la virgen, sector caracterizado principalmente por ser una zona residencial de estratos 2 y 3 . En esta zona se presentaron 44 hurtos de vehículos en el periodo de estudio, siendo el arma de fuego la herramienta de hurto más utilizada (65\%), seguido por el uso de armas blancas (20\%). De los 44 hurtos cometidos 40 corresponden a motocicletas.

ZCHV 5: Representa la zona del mercado público de la ciudad, la cual se caracteriza por la presencia intensiva de comercios en general, tráfico congestionado por la invasión de las vías públicas y las labores logísticas de los vehículos de carga, comprendiendo barrios tales como Santa Lucia, La esperanza, el Prado, Calamares, Zaragocilla, Escallón Villa, Chinquinquirá, Las Gaviotas, entre otros sectores aledaños Es la zona con mayor incidencia de hurto de vehículos, 284 en total. De los cuales el $60 \%$ corresponde a motocicletas, el $23 \%$ a automotores y el $17 \%$ a Camiones. En las particularidades de la zona se encuentra el alto uso de armas de fuego (61\%), además de elementos contundentes y cortopunzantes (27\%). Un hecho significativo de anotar es la alta frecuencia de ocurrencia de los hechos en los días laborables de la semana.

ZCHV 6: Representa la zona de frontera con el municipio de Turbaco, sector donde predomina las bodegas de uso industrial y logístico del sector de Ternera, igualmente existe una alta concentración de vivienda de estratos 2 y 3 . En esta zona la incidencia de los robos es baja, y el 100\% de los robos corresponde a motocicletas bajo la intimidación por arma de fuego.

ZCHV 7: Representa un sector densamente poblado de la ciudad, caracterizado por la presencia de Barrios subnormales, con poca infraestructura de vías publicas, transporte mobiliario urbano. Es la segunda zona con mayor número de hurtos, caracterizados estos por un $65 \%$ correspondientes a motocicletas, siendo el arma blanca y la intimidación por arma blanca el modo mas frecuente de operación del hurto.

\section{Conclusión}

Durante el desarrollo del presente estudio se lograron identificar variables claves que inciden sobre la percepción de seguridad de la ciudad, tales como la hora, zona específica del hurto, día de la semana y el tipo de arma empleada.

En otra aproximación, se aporta a la comunidad científica una estructura de pensamiento para estudiar el problema de hurto de vehículos de una ciudad utilizando la minería de datos. En los aspectos operativos, los resultados obtenidos favorecen una sofisticada intepretación de la problemática de los hurtos en Cartagena, al presentar los clústeres del delito en forma de Zona Caracteristicas de Hurto de Vehículos, sobre las cuales se pueden desarrollar procesos de planeación estratégica y operativa, considerando cada una de las particularidades de las zonas, en relación al objeto hurtado, el arma usada para ejecutar el robo.

Lo anterior, se plantea en primera instancia como un insumo informativo para optimizar el uso de los recursos de seguridad, reconociendo estos como factores escasos en las ciudades colombianas. 


\section{Referencias}

Abullarade, J. C. (2016). Criminalidad y Emprendimiento en Guatemala: 2016.

Cubel, F. M. (2016). El estado de la seguridad en América Latina 2015. bie3: Boletín IEEE, (1), 351-369.

Dammert, L., \& Arias, P. (2007). El desafío de la delincuencia en América Latina: Diagnóstico y respuestas de política. Seguridad y violencia: desafíos para la ciudadanía. FLACSO, 21-66.

De-La-Hoz, E. J., De-La-Hoz, E. J., \& Fontalvo, T. J. (2019). Metodología de Aprendizaje Automático para la Clasificación y Predicción de Usuarios en Ambientes Virtuales de Educación. Información tecnológica, 30(1), 247-254. https://doi.org/10.4067/S0718-07642019000100247

Fajardo Pascagaza, E. (2016). Hacia la caracterización de los valores democráticos y ciudadanos de las niñas y niños escolares: una mirada desde la filosofía para niños. Amauta, 14(27), 71-86. https://doi. org/10.15648/am.27.2016.7

Félix, J. Q., Ponce, R. A. C., Hernández, J. M. O., \& Llamas, R. V. (2015). Delincuencia y actividad económica en México. Norteamérica, 10(2), 187-209.

Flores Arias, P. (2014). Modelación y predicción de focos de criminalidad basado en modelos probabilísticos.

Fontalvo-Herrera, T. J., Delahoz, E. J., Mendoza-Mendoza, A. A., Fontalvo-Herrera, T. J., Delahoz, E. J., \& Mendoza-Mendoza, A. A. (2018). Aplicación de Minería de Datos para la Clasificación de Programas Universitarios de Ingeniería Industrial Acreditados en Alta Calidad en Colombia. Información tecnológica, 29(3), 89-96. https://doi.org/10.4067/S0718-07642018000300089

Güitron, A. C. M., \& Guerrero, L. R. V. (2017). Inseguridad pública y miedo al delito, un análisis de las principales perspectivas teóricas y metodológicas para su estudio. Letras Jurídicas, 6(6). Recuperado de http://letrasjuridicas.cuci.udg.mx/index.php/letrasjuridicas/article/view/63

Gutiérrez, J. (2013). Violencia juvenil urbana: Estrategias de políticas públicas en Nicaragua y El Salvador. Recuperado de https://repositorio.utdt.edu/handle/utdt/1466

Londoño, J. L., \& Guerrero, R. (1999). Violencia en América Latina: Epidemiología y Costos (Núm. 3074). Recuperado de Inter-American Development Bank, Research Department website: https://ideas.repec. org/p/idb/wpaper/3074.html

Oates, B. J. (2005). Researching information systems and computing. Sage.

Obrecht Ihl, P. (2014). Predicción de crimen usando modelos de Markov ocultos.

Peña Suárez, A. (2017). Modelo para la Caracterización del Delito en la Ciudad de Bogotá, Aplicando Técnicas de Minería de Datos Espaciales.

Ripoll, C. A. (2015). Plenitud de la Metafísica de la Alteridad. Amauta, 13(25), 197-206. Retrieved from http:// investigaciones.uniatlantico.edu.co/revistas/index.php/Amauta/article/view/1285

Ripollés, J. L. D. (2006). Algunos rasgos de la delincuencia en España a comienzos del Siglo XXI. Revista Española de Investigación Criminológica: REIC, (4), 1. Recuperado de https://dialnet.unirioja.es/servlet/ articulo?codigo $=2083343$

Rodriguez Rojas, L. A. (2017). Generación del Mapa de Crimen del Municipio de Zipaquira Mediante Herramientas Geoestadisticas y SIG.

Rojas Lopez, D. A., \& Ramirez, C. F. (2017). Análisis de la percepción del hurto a personas entre 2012 y 2015 en Bogotá.

Sánchez Salinas, O. A., \& Fuentes Flores, C. M. (2016). El robo de vehículos y su relación espacial con el 
contexto sociodemográfico en tres delegaciones centrales de la Ciudad de México (2010). Investigaciones geográficas, (89), 107-120.

Sani, A., \& Nunes, L. M. (2016). Diagnóstico de seguridad/inseguridad. Un estudio exploratorio en una comunidad urbana. Anuario de Psicología Jurídica, 26(1), 102-106.

Soto, J. (2017). Desde una ontología del lenguaje hacia una ética intercultural de la alteridad. Amauta, 15(30), 135-150. https://doi.org/10.15648/am.30.2017.10

Vázquez González, C., \& Soto Urpina, C. (2013). El análisis geográfico del delito y los mapas de la delincuencia.

Vizuete Galeas, D. A. (2013). Determinación de los lugares de mayor incidencia de delitos y violencia en el Distrito metropolitano de Quito con base en técnicas estadísticas espaciales. 